www.nature.com/ja

\title{
In vitro activity of two old antibiotics against clinical isolates of methicillin-resistant Staphylococcus aureus
}

\author{
Xu-Hong Yu ${ }^{1}$, Xiu-Jie Song ${ }^{2}$, Yun Cai ${ }^{1}$, Bei-Bei Liang ${ }^{1}$, De-Feng Lin $^{1}$ and Rui Wang ${ }^{1}$ \\ The objective of this paper was to investigate the in vitro effects of fusidic acid combined with fosfomycin against \\ methicillin-resistant Staphylococcus aureus (MRSA). In all, 196 MRSA strains isolated from three clinical specimens of human \\ infections from hospitals in China were used in this study. The checkerboard method was used to determine whether \\ combinations act synergistically against these strains. The susceptibility results for fusidic acid and fosfomycin were interpreted \\ according to the guidelines of the Clinical and Laboratory Standards Institute. The combination of fusidic acid and fosfomycin \\ demonstrated the following interactions: $87.76 \%(172 / 196)$ synergism, $12.24 \%(24 / 196)$ indifference and no antagonism was \\ seen (minimum and maximum fractional inhibitory concentration index 0.14 and 0.75 , respectively). Thus, combinations of \\ fusidic acid and fosfomycin show synergism for most of the MRSA isolates tested in this study, and may be a future therapeutic \\ alternative for infections caused by MRSA.
}

The Journal of Antibiotics (2010) 63, 657-659; doi:10.1038/ja.2010.105; published online 29 September 2010

Keywords: fosfomycin; fractional inhibitory concentration (FIC); fusidic acid; methicillin-resistant Staphylococcus aureus (MRSA); synergism

\section{INTRODUCTION}

It is well known that methicillin-resistant Staphylococcus aureus (MRSA) causes life-threatening infections, such as meningitis, bone marrow destruction, sepsis, endocarditis, septic pulmonary emboli and extensive soft tissue infection in humans; MRSA has become the most prevalent pathogen worldwide. ${ }^{1,2}$ Currently, over $60 \%$ of S. aureus isolates from intensive care units in the USA and over $50 \%$ of isolates from inpatient non-intensive care unit hospital settings are caused by MRSA. ${ }^{3,4}$ In Asia, MRSA is highly endemic in health care settings with prevalence rates of $>50 \%$ in many hospitals. In Hong Kong, MRSA is known to be endemic in hospitals since mid 1980s. ${ }^{5}$ Vancomycin is an effective agent in treating serious infections due to MRSA. ${ }^{6}$ However, other possibilities should be explored for those patients unable to tolerate the use of this drug or in the case of development of resistance to vancomycin among the strains of MRSA. $^{7}$ In recent years, new antistaphylococcal antibiotics, such as linezolid, daptomycin, tygecicline or telavancin, have been developed, but their cost is high. The infections caused by MRSA are serious and are difficult to treat. Therefore, we attempted to find an effective and safe combination of antibiotics to treat the infections caused by MRSA. A number of old antibiotic compounds, such as fosfomycin and fusidic acid, are reemerging as valuable alternatives for the treatment of difficult-to-treat infections. ${ }^{8}$

Fusidic acid has a high degree of activity against $S$. aureus, including MRSA. $^{9-11}$ However, the development of resistance during treatment with fusidic acid when this antibiotic is used alone has been reported. ${ }^{5,12,13}$ Resistance to fusidic acid can be produced readily in the laboratory by growing $S$. aureus in the presence of increasing concentrations of this antibiotic. ${ }^{14}$

Fosfomycin is a structurally unique antibiotic that is chemically unrelated to any other antimicrobial agent. Fosfomycin is a phosphoenolpyruvate analog that irreversibly inhibits enolpyruvate transferase resulting in the prevention of formation of $\mathrm{N}$-acetyl-muramic acid, the first stage of peptidoglycan synthesis of the bacterial cell wall. ${ }^{15,16}$ Fosfomycin is bactericidal against MRSA and has also been reported to directly interact with the immune system by affecting $\mathrm{T}$ lymphocyte function. ${ }^{17}$

The objective of this study was to evaluate the in vitro effects of fusidic acid combined with fofomycin upon MRSA strains.

\section{MATERIALS AND METHODS}

\section{Bacterial isolates}

We collected and evaluated 196 methicillin-resistant S. aureus strains from three Chinese hospitals (PLA General Hospital 98 strains, Beijing Hospital 33 strains and Peking Union Medical College Hospital 65 strains) in this study. This isolates were collected from the blood (29\%), nose (7\%), sputum $(21 \%)$, wound sites (15\%), skin (23\%), urine (3\%), unspecified (3\%) of the patients. All the strains were identified by VITEK-2 system (Biomerieux, Marcy l'Etoile, France). S. aureus American type culture collection 25923 was used for quality control in the latex agglutination test. SLIDEX Staph Plus (Biomerieux, France) is a rapid latex agglutination test for the identification of $S$. aureus strains. Disk agar diffusion method (Kirby-Bauer) was used to test susceptibility. Broth dilution testing was used for determining in vitro effects of fusidic acid combined with fosfomycin. S. aureus ATCC 29213 was used as quality control strain for these tests. ${ }^{18}$ 


\section{Antimicrobials}

All antibiotics were obtained from the National Institute for the control of Pharmaceutical and Biological Products (Beijing, China). The purity of fusidic acid was $96 \%$ and fosfomycin $99 \%$. Antibiotic powders were used to prepare stock solutions at concentrations of $1024 \mu_{\mathrm{g} \mathrm{ml}}^{-1}$ as recommended by the Clinical and Laboratory Standards Institute. ${ }^{18}$

\section{Minimum inhibitory concentration (MIC) determination and synergy testing}

Initially, all the isolates were tested against a single compound using the broth microdilution method according to Clinical and Laboratory Standards Institute standards. Synergy tests were performed in 96-well broth microdilution plates, containing two antimicrobial agents in twofold dilutions dispensed in a checkerboard format. ${ }^{19}$

Fosfomycin was dispended alone in the first row with concentration ranging from 0.5 to $256 \mu \mathrm{g} \mathrm{ml}^{-1}$, whereas fusidic acid was dispended in the first column with concentration ranging from 0.03 to $2 \mu \mathrm{g} \mathrm{m}^{-1}$. For seven strains, fusidic acid concentration ranging from 0.25 to $16 \mu \mathrm{g} \mathrm{ml}^{-1}$ was used. The concentrations were set according to the MIC values obtained in a preliminary test. Inocula were prepared by suspending growth from agar plates in MuellerHinton broth to a density of $0.5 \mathrm{McF}$ arland standard and were diluted 1:10 to produce a final inoculum of $1.5 \times 10^{5}$ colony-forming units per $\mathrm{ml}$ with a multipoint inoculators. The trays were incubated aerobically overnight. Standard quality control strains were incubated with each run. Interpretations of the antimicrobial combinations were based on calculation of the fractional inhibitory concentration index (FICI) for each drug pair. The FICI was calculated as the MIC of drug A and B in combination divided by the MIC of drug A or B alone, according to the following formula: ${ }^{20,21}$

$$
\begin{aligned}
\text { FICI }= & (\text { MIC of DrugA in combination } / \text { MIC of DrugA alone }) \\
& +(\text { MIC of the DrugB in combination } / \text { MIC of the DrugB alone })
\end{aligned}
$$

\begin{tabular}{ll}
\hline $\mathrm{FICl} \leqslant 0.5$ & Synergism \\
$0.5<\mathrm{FICl} \leqslant 4$ & Indifferent \\
$\mathrm{FICl}>4$ & Antagonistic
\end{tabular}

\section{Statistical analysis}

Statistical analysis was performed by using the SAS statistical program.

\section{RESULTS}

MIC for fusidic acid combined with fosfomycin against MRSA

The $\mathrm{MIC}_{50}$ and $\mathrm{MIC}_{90}$ for the strains tested in combination, respectively, were fusidic acid 0.03 and $0.125 \mu \mathrm{g} \mathrm{ml}^{-1}$, and for fosfomycin 8 and $16 \mu \mathrm{g} \mathrm{ml}^{-1}$, respectively, whereas alone they were fusidic acid $0.125 / 0.5 \mu \mathrm{g} \mathrm{ml}^{-1}$ and fosfomycin $64 / 128 \mu \mathrm{g} \mathrm{ml}^{-1}$, respectively. Fusidic acid MIC values for $9.2 \%$ of the strains were $>1 \mu \mathrm{g} \mathrm{ml}^{-1}$, whereas fosfomycin MIC values for $66.8 \%$ of the strains were $\geqslant 64 \mu \mathrm{g} \mathrm{ml}^{-1}$ (Table 1). However, when they were combined fusidic acid MIC values for $99.7 \%$ of the strains were $\leqslant 1 \mu \mathrm{g} \mathrm{ml}^{-1}$. Although fosfomycin MIC values for all of the strains were $\leqslant 32 \mu \mathrm{g} \mathrm{ml}^{-1}$.

Table 1 Minimum inhibitory concentration (MIC) (in $\mu \mathrm{g} \mathrm{ml}^{-1}$ ) of fusidic acid combined with fosfomycin in Staphylococcus aureus $(N=196)$

\begin{tabular}{lcccccccc}
\hline & \multicolumn{3}{c}{ Single } & & \multicolumn{3}{c}{ Combination } \\
\cline { 2 - 4 } \cline { 6 - 8 } Antimicrobial & MIC & MIC & MIC range & & MIC & MIC & M0 & MIC range \\
\hline Fusidic acid & 0.125 & 0.5 & $0.03-8$ & & 0.0300 & 0.125 & $0.0008-2$ \\
Fosfomycin & 64 & 128 & $8-256$ & 8 & 16 & $0.5-32$ \\
\hline
\end{tabular}

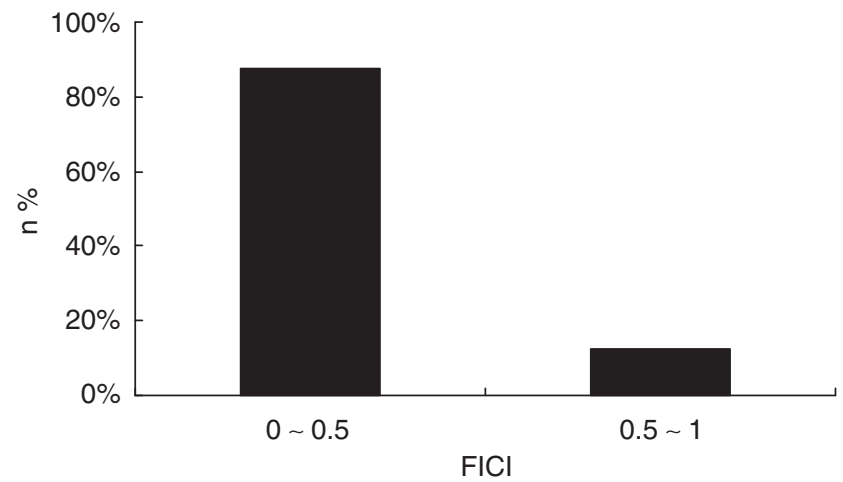

Figure 1 Fractional inhibitory concentration index (FICl) of fusidic acid combined with fosfomycin in methicillin-resistant Staphylococcus aureus $(N=196)$.

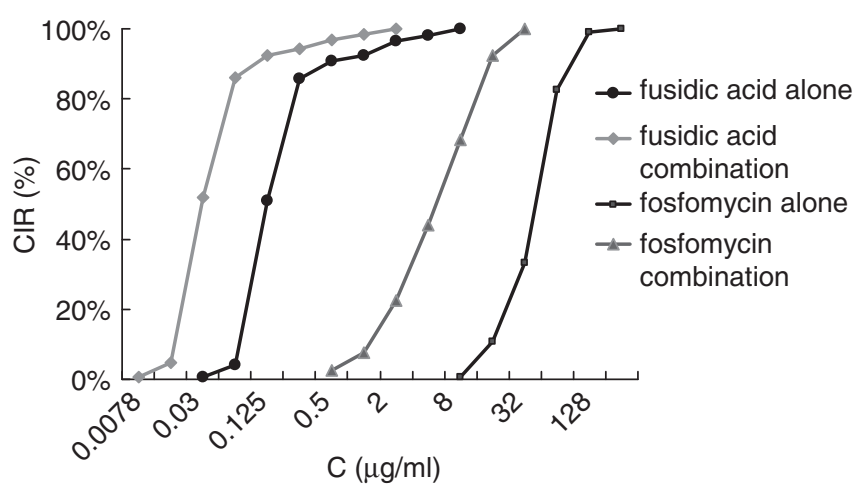

Figure 2 The cumulative inhibition ratio (CIR) of fusidic acid and/or fosfomycin against methicillin-resistant Staphylococcus aureus $(N=196)$. C, concentration.

FICI of fusidic acid combined with fosfomycin for MRSA Of the strains tested, 172 isolates $(87.75 \%)$ showed synergistic interaction $(\mathrm{FICI} \leqslant 0.5)$ and 24 isolates $(12.25 \%)$ showed indifference $(0.5<$ FICI $<1$ ) (Figure 1$)$. No antagonism (minimum and maximum fractional inhibitory concentration index 0.14 and 0.75 , respectively) was observed.

The cumulative inhibition ratio of fusidic acid and/or fosfomycin against MRSA

Cumulative inhibition ratio of fusidic acid and/or fosfomycin against MRSA are shown in Figure 2. The curve of the cumulative inhibition ratio was moved to the left when the two drugs were used together, which suggested the potential utility of this combination.

\section{DISCUSSION}

MRSA has reemerged as a global public health problem. ${ }^{12}$ Over the last decade, MRSA has become increasingly common in hospitals and in community settings. ${ }^{22-24}$ Combination antimicrobial is an important treatment approach for infections. Synergy is one of the most common reasons for using combination antimicrobial therapy. Thus, the search for combinations of antibiotics might yield more effective treatment options and fewer side effects.

Some compounds are unable to inhibit or kill bacteria by themselves, but can block bacterial mechanisms of resistance, enhancing the activity of other antimicrobials administered in combination. As the emergence of resistance has been associated with monotherapy, ${ }^{25}$ some authors found that fusidic acid in combination with linezolid 
avoided the appearance of resistance to the partner drug. ${ }^{26}$ Some data suggests a synergistic activity of fosfomycin with various antistaphylococcal agents. ${ }^{16}$ Moreover, fosfomycin is far less expensive than linezolid. ${ }^{25}$ Although fosfomycin has limited in vitro activity against MRSA, our data suggests its combination with fusidic acid is synergistic against MRSA. Moreover, fosfomycin has the advantage of low toxity and high safety.

In our study using 196 MRSA isolates from hospitals in China, fusidic acid combined with fosfomycin was synergistic with $87.76 \%$ of strains; $12.24 \%$ showed indifference and no antagonistic were observed. Most of the isolates evaluated in the study were intermediate susceptible or resistant to fusidic acid and fosfomycin. Most of the isolates had MIC values within the susceptible range for fusidic acid, whereas only $66.8 \%$ for fosfomycin. However, in combinations, fusidic acid MIC values for $99.7 \%$ of the strains were $\leqslant 1 \mathrm{ml}^{-1}$, whereas fosfomycin MIC values for all of the strains were $\leqslant 32 \mu \mathrm{g} \mathrm{ml}^{-1}$. From our in vitro results, the combination of fusidic acid with fosfomycin is active at lower concentrations than the single agents. This combination could also prevent the emergence of resistance to current antistaphylococcal drugs. Thus fosfomycin and fusidic acid should be used in combination when treating MRSA infections to prevent the emergence of resistance to either of these antibiotics. From the cumulative inhibition ratio curve we can still see the notable difference when fusidic acid and fosfomycin were tested in combination, supporting the combination as a future therapeutic alternative provided that pharmacokinetics could be optimized for both drugs.

In conclusion, combinations of fusidic acid with fosfomycin have significant synergistic activity against most of the strains of MRSA that were tested. The presence of fofomycin and fusidic acid is crucial to prevent further emergence of these resistant strains. For this reason, the combinations may be useful in the treatment of infections caused by MRSA. Further studies, especially animal models will be required to establish if the in vitro synergistic activity observed with fosfomycin and fusidic acid is also evident in vivo.

\section{ACKNOWLEDGEMENTS}

The project was supported by the National Natural Science Foundation of China (no. 30873127).

1 Thornsberry, C. Epidemiology of staphylococcal infections-a USA perspective. J. Chemother. 6(Suppl 2), 61-65 (1994).

2 Vandenbroucke-Grauls, C. Epidemiology of staphylococcal infections-a European perspective. J. Chemother. 6(Suppl 2), 67-70 (1994).

3 Centers for Disease Control and Prevention (CDC). MRSA among ICU Patients, (1995-2004).
4 National Nosocomial Infections Surveillance (NNIS) System Report, data summary from January 1992 through June 2004, issued October Am. Infect. Control 32, 470-485 (2004).

5 Ho, P. L., Yuen, K., Yam, W., Sai-yin, W. S. \& Luk, W. Changing patterns of susceptibilities of blood, urinary and respiratory pathogens in Hong Kong. J. Hosp. Infect. 31, 305-317 (1995).

6 Craven, D. E., Kollisch, N. R., Hseih, C. R., Conolly, M. G. \& McCabe, W. R. Vancomycin treatment of bacteremia caused by oxacillin-resistant Staphylococcus aureus: comparison with beta-lactam antibiotic treatment of bacteremia caused by oxacillin-sensitive Staphylococcus aureus. J. Infect. Dis. 147, 137-143 (1983).

7 Sorrell, T. C., Packham, D. R., Shanker, S., Foldes, M. \& Munro, R. Vancomycin therapy for methicillin-resistant Staphylococcus aureus. Ann. Intern. Med. 97, 3440-3501 (1982).

8 Falagas, M. E., Grammatikos, A. P. \& Michalopoulos, A. Potential of old-generation antibiotics to address current need for new antibiotics. Expert. Rev. Anti. Infect. Ther. 6, 593-600 (2008).

9 Brown, E. M. \& Thomas, P. Fusidic acid resistance in Staphylococcus aureus isolates. Lancet 359, 803 (2002).

10 Chang, S. C., Hsieh, S. M., Chen, M. L., Sheng, W. H. \& Chen, Y. C. Oral fusidic acid fails to eradicate methicillin-resistant Staphylococcusaureus colonization and results in emergence of fusidic acid-resistant strains. Diagn. Microbiol. Infect. Dis. 36, 131-136 (2000).

11 Ravenscroft, J. C., Layton, A. \& Barnham, M. Observations on high levels of fusidic acid resistant Staphylococcus aureus in Harrogate, North Yorkshire, UK. Clin. Exp. Dermatol. 25, 327-330 (2000).

12 Grundmann, H., Ires-de-Sousa, M. \& Boyce, J. Emergence and resurgence of methicillin-resistant Staphylococcus aureus as a public-health threat. Lancet 368, 874-885 (2006).

13 Maviglia, R., Nestorini, R. \& Pennisi, M. Role of old antibiotics in multidrug resistant bacterial infections. Curr. Drug. Targets 10, 895-905 (2009).

14 O’Brien, F. G., Botterill, C. I., Endersby, T. G., Lim, R. L. G., Grubb, W. B. \& Gustafson, J. E. Heterogeneous expression of fusidic acid resistance in Staphylococcus aureus with plasmid or chromosomally encoded fusidic acid resistance genes. Pathology 30, 299-303 (1998).

15 Forsgen, A. \& Walder, M. Antimicrobial activity of fosfomycin in vitro. J. Antimicrob. Chemother. 11, 467-471 (1983).

16 Grif, K., Dierich, M. P., Pfaller, K., Miglioli, P. A. \& Allerberger, F. In vitro activity of fosfomycin in combination with various antistaphylococcal substances. J. Antimicrob. Chemother. 48, 209-217 (2001).

17 Morikawa, K., Oseko, F., Morikawa, S. \& Sawada, M. Immunosuppressive activity of fosfomycin on human T-lymphocyte function in vitro. Antimicrob. Agents Chemother. 37, 2684-2687 (1993).

18 National Committee for Clinical Laboratory Standards (NCCLS). Performance standards for antimicrobial susceptibility testing. Information Supplement M100, S19 (2009).

19 Moody, J. Synergism testing: broth microdilution checkerboard and broth macrodilution method. in Clinical Microbiology Procedures Handbook (ed. Isenberg, H. D.) 2nd edn, 1-28 (ASM Press: Washington, DC, 2004).

20 Carmona, P., Romá, E., Monte, E., García, J. \& Gobernado, M. Papel de linezolid en terapéutica antimicrobiana. Enferm. Infecc. Microbiol. Clin. 21, 30-41 (2003).

21 Gobernado, M. Fosfomicina. Rev. Esp. Quimioterap. 16, 15-40 (2003).

22 Moreno, F., Crisp, C. \& Jorgensen, J. H. Methicillin-resistant Staphylococcus aureus as a community organism. Clin. Infect. Dis. 21, 1308-1312 (1995).

23 Panlilio, A. L., Culver, D. H. \& Gaynes, R. P. Methicillin-resistant Staphylococcus aureus in US hospitals, 1975-1991. Infect. Control Hosp. Epidemiol. 13, 582-586 (1992).

24 Robinson, D. A., Kearns, A. M. \& Holmes, A. Re-emergence of early pandemic Staphylococcus aureus as a community-acquired methicillin-resistant clone. Lancet 365, 1256-1258 (2005).

25 Whitby, M. Fusidic acid in the treatment of methicillin-resistant Staphylococcus aureus. Int. J. Antimicrob. Agents 12, S67-S71 (1999).

26 Grohs, P., Kitzis, M. D. \& Gutmann, L. In vitro bactericidal activities of linezolid in combination with vancomycin, gentamicin, ciprofloxacin, fusidic acid, and rifampin against Staphylococcus aureus. Antimicrob. Agents Chemother. 47, 418-420 (2003). 